\title{
Research on the Influence of Switching Frequency on Low-Frequency Oscillation in the Voltage-Controlled Buck-Boost Converter
}

\author{
Faqiang Wang and Xikui Ma \\ The State Key Laboratory of Electrical Insulation and Power Equipment, School of Electrical Engineering, \\ Xi'an Jiaotong University, Xi'an 710049, China \\ Correspondence should be addressed to Faqiang Wang, eecjob@126.com
}

Received 21 January 2011; Revised 25 April 2011; Accepted 19 May 2011

Academic Editor: Oded Gottlieb

Copyright (C) 2011 F. Wang and X. Ma. This is an open access article distributed under the Creative Commons Attribution License, which permits unrestricted use, distribution, and reproduction in any medium, provided the original work is properly cited.

\begin{abstract}
The influence of switching frequency on the low-frequency oscillation in the voltage-controlled buck-boost converter is studied in this paper. Firstly, the mathematical model of this system is derived. And then, a glimpse at the influence of switching frequency on the low-frequency oscillation in this system by MATLAB/Simulink is given. The improved averaged model of the system is established, and the corresponding theoretical analysis is presented. It is found that the switching frequency has an important influence on the low-frequency oscillation in the system, that is, the low-frequency oscillation is easy to occur when the switching frequency is low. Finally, the effectiveness of the improved averaged model and the theoretical analysis are confirmed by circuit experiment.
\end{abstract}

\section{Introduction}

Unlike the PWM-controlled boost converter and the PWM-controlled buck converter, if the input voltage polarity of the PWM-controlled buck-boost converter is positive, its output voltage polarity will be negative [1], and accordingly this converter has been widely used in the condition that the negative output voltage is needed, such as the audio amplifier, the signal generator, or the data transmission interface. Therefore, the modeling, dynamical behaviors of the PWM-controlled buck-boost converter has been a focal topic research in recent years [2-11]. Especially, due to the fact that the PWM-controlled buck-boost converter is a strong nonlinear and nonautonomous system, the various kinds of nonlinear phenomena can be observed and the theoretical analysis of these nonlinear phenomena have also attracted much attention of researchers [3-11]. For example, Aroudi et al. studied the low-frequency oscillation and chaos in the voltage-controlled buck-boost converter by using the discrete model in 2000 [3]. Wu et al. studied the subharmonic and chaos in 
the current-controlled buck-boost converter by using the discrete model in $2002[4,5]$. From the existing literatures, it is found that the main analysis model for investigating the nonlinear phenomenon in the PWM-controlled buck-boost converter is the discrete model [3-11]. However, this model is too complicated, especially for obtaining the solution of each state of the PWM-controlled buck-boost converter. Thus, it is difficult to use the discrete model to analyze and design the PWM-controlled buck-boost converter in the engineering applications. Especially, if the low-frequency region of the PWM-controlled buck-boost converter is only concerned, the discrete model is too redundant [12]. Additionally, the conventional averaged model is widely accepted to analyze and design this converter. Also, the conventional averaged model can be used to identify the occurrence of the low-frequency oscillation, and this point has been confirmed by Iu and Tse in [13]. However, the influence of the switching frequency on the low-frequency oscillation in the PWM-controlled buckboost converter cannot be identified since there is no switching frequency parameter in its conventional averaged model. Therefore, it is necessary to explore other models to analyze the influence of the switching frequency on the low-frequency oscillation in the PWMcontrolled buck-boost converter effectively and successfully.

In this paper, the influence of the switching frequency on the low-frequency oscillation in the voltage-controlled buck-boost converter is analyzed by establishing the improved averaged model. The rest of the paper is organized as follows. In Section 2, the circuit operation of the system and its mathematical model are given. In Section 3, a glimpse at the low-frequency oscillation in the voltage-controlled buck-boost converter from the MATLAB/Simulink is presented. And then, the improved averaged model of the system is established. In Section 5, theoretical analysis of the influence of switching frequency on the low-frequency oscillation in the system is given. In Section 6, some experimental results are given to confirm the effectiveness of the theoretical analysis. Finally, some concluding remarks and comments are given in Section 7.

\section{Circuit Operation and Its Mathematical Model}

The schematic of the voltage-controlled buck-boost converter is shown in Figure 1. Obviously, this system consists of two parts: the buck-boost converter and the voltage controller. Since the polarity of the output voltage is negative, the inverting amplifier $\left(\mathrm{AM}_{1}\right)$ should be inserted in the voltage controller loop. Note that $v_{0}$ is the output voltage of the voltagecontrolled buck-boost converter, $i_{L}$ is the current through of the inductor $L, v_{\mathrm{vf}}$ is the output voltage of the voltage compensator, $d$ is the duty cycle, $V_{\text {in }}$ is the input voltage of the system, $R$ is the load resistor, $V_{\text {ref }}$ is the reference voltage, and $C_{\mathrm{vf}}, R_{\mathrm{vf}}, R_{\mathrm{vi}}$, and $R_{\mathrm{vd}}$ are the parameters of the voltage controller.

According to the circuit theory and the characteristic of each component, the mathematical model of the voltage-controlled buck-boost converter in the following three states can be derived.

The first state ( $Q$ is on and $D$ is off):

$$
\begin{gathered}
\frac{d i_{L}}{d t}=\frac{V_{\text {in }}}{L}, \\
\frac{d v_{0}}{d t}=-\frac{v_{0}}{R C^{\prime}} \\
\frac{d v_{\mathrm{vf}}}{d t}=\frac{R_{\mathrm{vf}}}{2 R_{\mathrm{vi}}+R_{\mathrm{vd}}}\left(-\frac{v_{0}}{R C}+\frac{v_{0}}{C_{\mathrm{vf}} R_{\mathrm{vf}}}\right)+\frac{V_{\mathrm{ref}}}{C_{\mathrm{vf}} R_{\mathrm{vd}}} .
\end{gathered}
$$




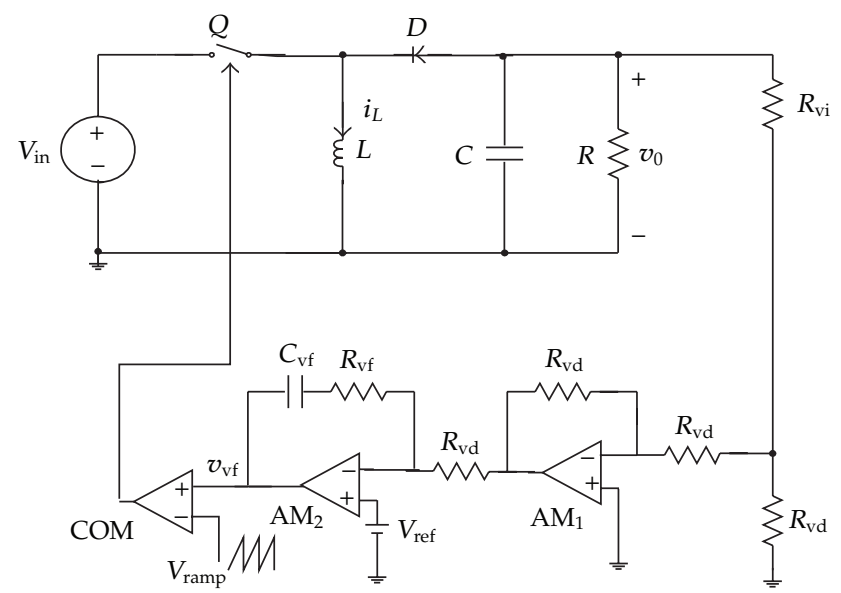

Figure 1: The schematic of the voltage-controlled buck-boost converter.

The second state ( $Q$ is off and $D$ is on):

$$
\begin{gathered}
\frac{d i_{L}}{d t}=\frac{v_{0}}{L}, \\
\frac{d v_{0}}{d t}=-\frac{i_{L}}{C}-\frac{v_{0}}{R C^{\prime}} \\
\frac{d v_{\mathrm{vf}}}{d t}=\frac{R_{\mathrm{vf}}}{2 R_{\mathrm{vi}}+R_{\mathrm{vd}}}\left(-\frac{i_{L}}{C}-\frac{v_{0}}{R C}+\frac{v_{0}}{C_{\mathrm{vf}} R_{\mathrm{vf}}}\right)+\frac{V_{\mathrm{ref}}}{C_{\mathrm{vf}} R_{\mathrm{vd}}} .
\end{gathered}
$$

The third state ( $Q$ is off and $D$ is off)

$$
\begin{gathered}
i_{L}=0, \\
\frac{d v_{0}}{d t}=-\frac{v_{0}}{R C^{\prime}} \\
\frac{d v_{\mathrm{vf}}}{d t}=\frac{R_{\mathrm{vf}}}{2 R_{\mathrm{vi}}+R_{\mathrm{vd}}}\left(-\frac{v_{0}}{R C}+\frac{v_{0}}{C_{\mathrm{vf}} R_{\mathrm{vf}}}\right)+\frac{V_{\mathrm{ref}}}{C_{\mathrm{vf}} R_{\mathrm{vd}}} .
\end{gathered}
$$

Note that the switch $Q$ is on or is off is determined by comparing the voltage $v_{\mathrm{vf}}$ with

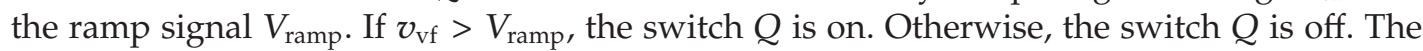
$V_{\text {ramp }}$ is described as

$$
V_{\text {ramp }}=V_{L}+\left(V_{U}-V_{L}\right)\left(\frac{t}{T} \bmod 1\right)
$$

where $V_{L}$ is the lower threshold, $V_{U}$ is the upper threshold, $V_{m}=V_{U}-V_{L}$ is the peak-to-peak value of the $V_{\text {ramp }}$, and $T$ is the switching period. 


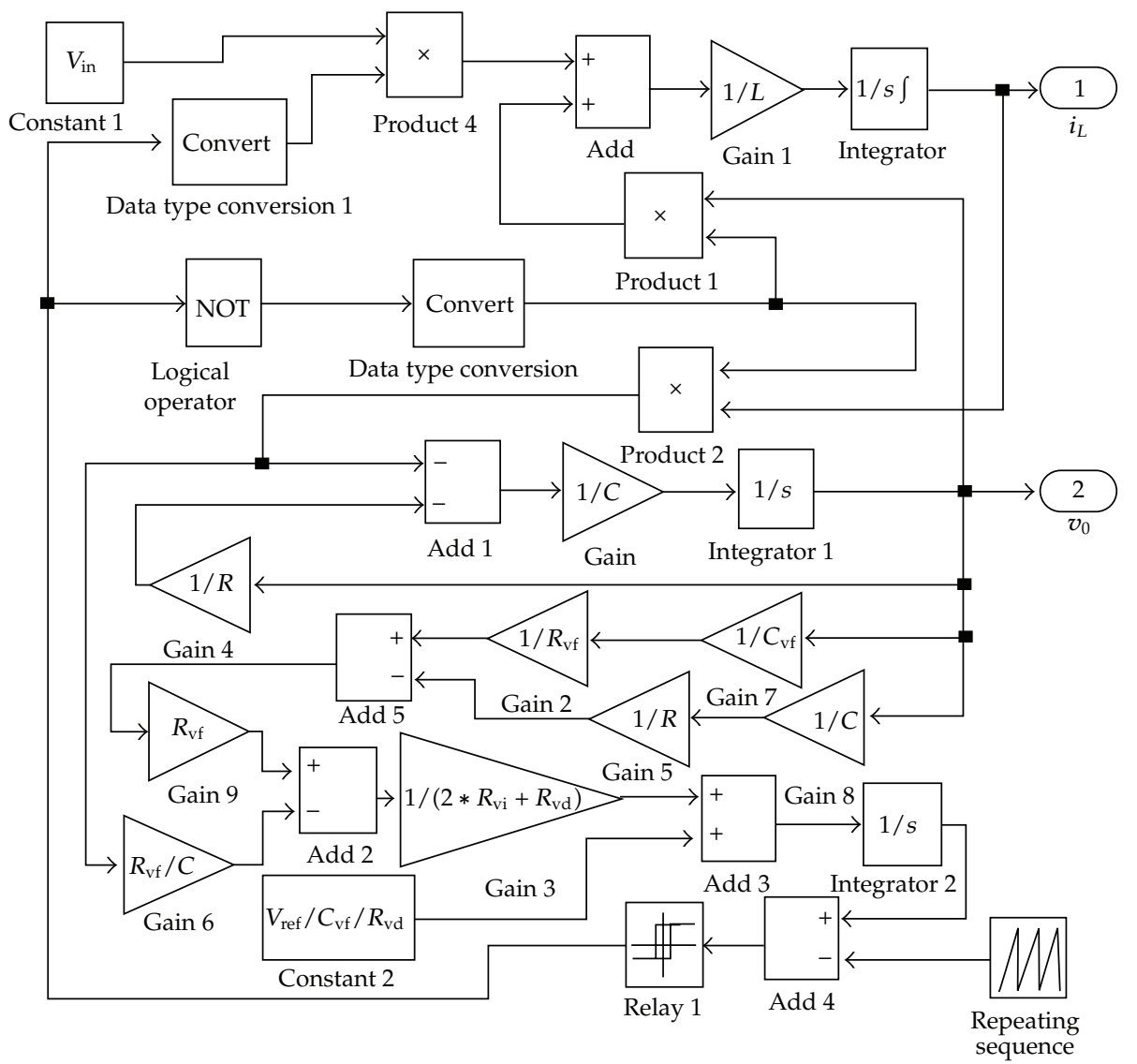

Figure 2: Simulation model for the system in the MATLAB/Simulink.

\section{A Glimpse at the Low-Frequency Oscillation from the MATLAB/Simulink}

According to (2.1)-(2.4), the simulation model for the voltage-controlled buck-boost converter in the MATLAB/Simulink is established and shown in Figure 2.

The circuit parameters are chosen as $V_{\text {in }}=12 \mathrm{~V}, L=3 \mathrm{mH}, C=10 \mu \mathrm{F}, R=100 \Omega$, $R_{\mathrm{vi}}=100 \mathrm{k} \Omega, R_{\mathrm{vd}}=20 \mathrm{k} \Omega, R_{\mathrm{vf}}=15 \mathrm{k} \Omega, C_{\mathrm{vf}}=1 \mu \mathrm{F}, V_{\text {ref }}=2 \mathrm{~V}, V_{L}=0 \mathrm{~V}, V_{U}=5 \mathrm{~V}$. The dynamical behaviors of the voltage-controlled buck-boost converter under different switching frequency are investigated as follows. Note that the MATLAB/Simulink is an effective and convenient tool for calculating the numerical solution of the system and it has been widely used in power electronics, such as DC-DC converter [14, 15], AC-DC converter [15], and matrix converter [16]. Furthermore, when the simulation process is carried out, the solver is chosen as Ode23tb.

The simulation results for $f=20 \mathrm{kHz}$ are shown in Figure 3. Figure 3(a) shows the time-domain waveform of the output voltage $v_{0}$ and Figure $3(\mathrm{~b})$ shows the time-domain waveform of the inductor current $i_{L}$. It is obvious that the system is in stable operation and the peak value of inductor current is equal to $0.673 \mathrm{~A}$, that is, $i_{\mathrm{LM}}=0.673 \mathrm{~A}$. 


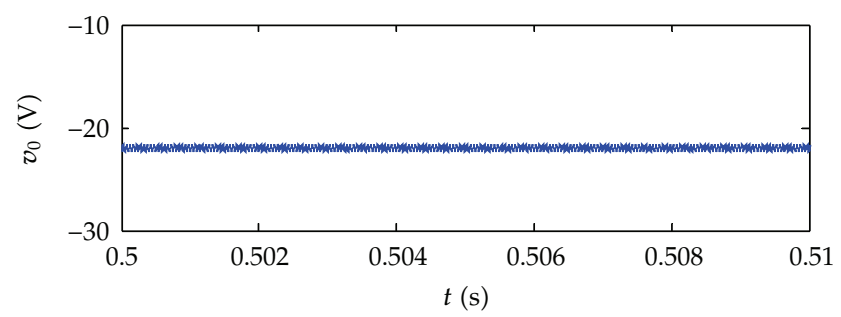

(a)

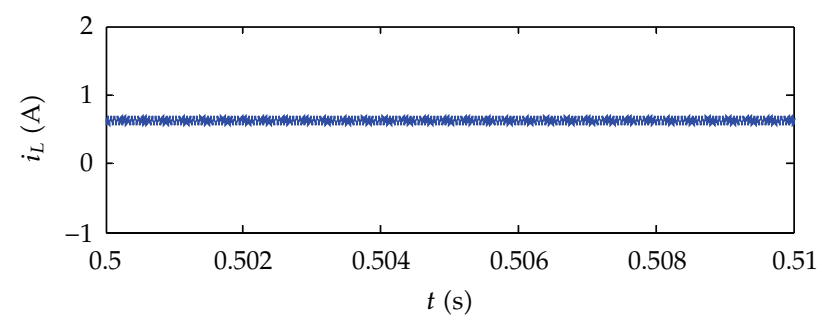

(b)

Figure 3: The MATLAB/Simulink simulation results for $f=20 \mathrm{kHz}(\mathrm{a}) v_{0}$, (b) $i_{L}$.

The simulation results for $f=4 \mathrm{kHz}$ is shown in Figure 4 . It is obvious that the system is in unstable operation and the low-frequency oscillation occurs. Moreover, the peak value of inductor current is equal to $1.294 \mathrm{~A}$, that is, $i_{\mathrm{LM}}=1.294 \mathrm{~A}$, which is about to two times larger than the one for $f=20 \mathrm{kHz}$ in stable operation. Obviously, the current stress of the switch and the diode is increased when the low-frequency oscillation occurs.

Also, in order to observe the influence of the switching frequency on the dynamical behaviors of the voltage-controlled buck-boost converter more clearly, the critical points of the occurrence of the low-frequency oscillation in the different parameter spaces from the MATLAB/Simulink are noted down and plotted in Figures 5 and 6. Figure 5 shows the results in the parameter space of the switching frequency $f$ versus the resistor $R_{\mathrm{vf}}$ and it is found that the low-frequency oscillation is easy to occur when the switching frequency $f$ is low or the resistor $R_{\mathrm{vf}}$ is high. Figure 6 shows the results in the parameter space of the switching frequency $f$ versus the capacitor $C$ and it is found that the low-frequency oscillation is easy to occur when the switching frequency $f$ is low or the capacitor $C$ is high.

From the above MATLAB/Simulink simulation results, the conclusion can be obtained that the switching frequency has an important influence on the occurrence of the lowfrequency oscillation in the voltage-controlled buck-boost converter, that is, the lowfrequency oscillation is easy to occur when the switching frequency is low, and accordingly it is necessary to explore the proper analysis model to investigate this influence in theoretic.

\section{Theoretical Derivation}

From Figures 3 and 4, one can see that the voltage-controlled buck-boost converter mainly operates in continuous conduction mode (CCM) operation, and accordingly only the $\mathrm{CCM}$ operation in the voltage-controlled buck-boost converter is considered in this paper. 


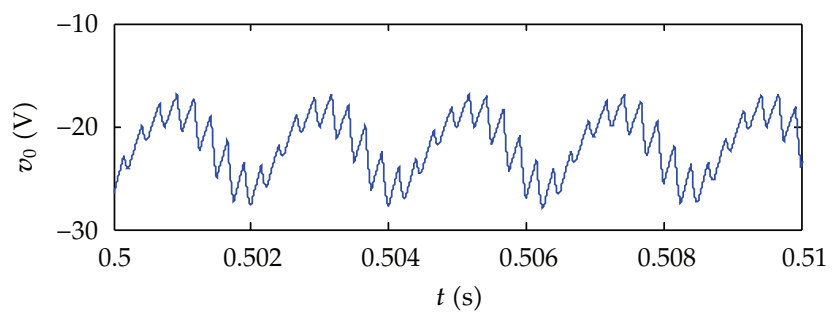

(a)

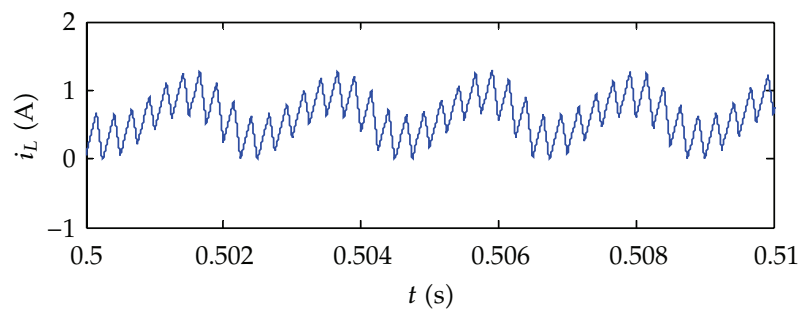

(b)

Figure 4: The MATLAB/Simulink simulation results for $f=4 \mathrm{kHz}(\mathrm{a}) v_{0}$, (b) $i_{L}$.

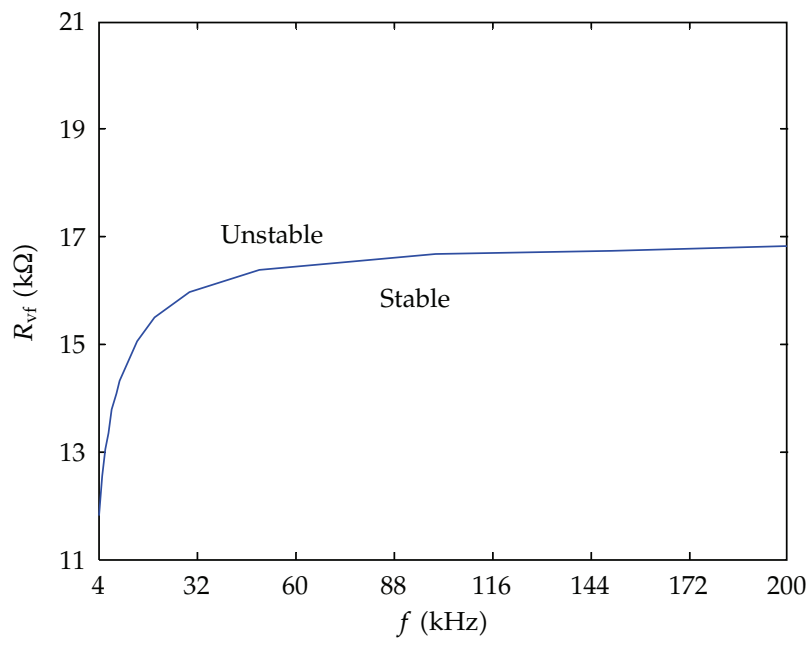

Figure 5: The boundaries in the parameter space of $f$ versus $R_{\mathrm{vf}}$ from the MATLAB/Simulink.

According to (2.1), (2.2), (2.4), and the average modeling method [13], the averaged model of the voltage-controlled buck-boost converter can be described directly as follows:

$$
\begin{gathered}
\frac{d\left\langle i_{L}\right\rangle}{d t}=\frac{\left\langle v_{0}\right\rangle}{L}(1-\langle d\rangle)+\frac{V_{\mathrm{in}}}{L}\langle d\rangle, \\
\frac{d\left\langle v_{0}\right\rangle}{d t}=-\frac{\left\langle i_{L}\right\rangle}{C}(1-\langle d\rangle)-\frac{\left\langle v_{0}\right\rangle}{R C}, \\
\frac{d\left\langle v_{\mathrm{vf}}\right\rangle}{d t}=\frac{R_{\mathrm{vf}}}{2 R_{\mathrm{vi}}+R_{\mathrm{vd}}}\left(-\frac{\left\langle i_{L}\right\rangle}{C}(1-\langle d\rangle)-\frac{\left\langle v_{0}\right\rangle}{R C}+\frac{\left\langle v_{0}\right\rangle}{C_{\mathrm{vf}} R_{\mathrm{vf}}}\right)+\frac{V_{\mathrm{ref}}}{C_{\mathrm{vf}} R_{\mathrm{vd}}} .
\end{gathered}
$$




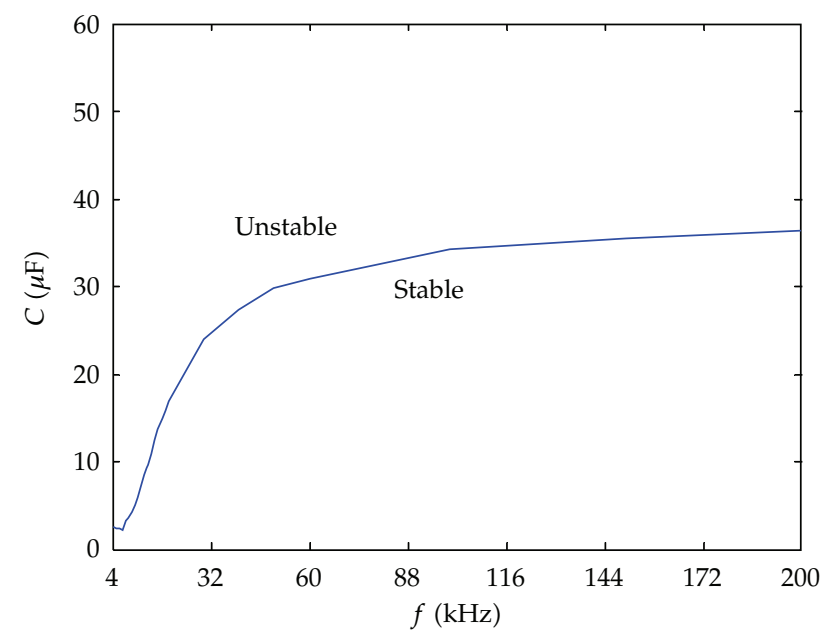

Figure 6: The boundaries in the parameter space of $f$ versus $C$ from the MATLAB/Simulink.

Note that $\left\langle i_{L}\right\rangle,\left\langle v_{0}\right\rangle,\left\langle v_{\mathrm{vf}}\right\rangle$, and $\langle d\rangle$ are the average value of $i_{L}, v_{0}, v_{\mathrm{vf}}$, and $d$, respectively. For the conventional averaged model, the duty cycle $\langle d\rangle$ is determined by the value $\left\langle v_{\mathrm{vf}}\right\rangle / V_{m}$ and this point leads to the fact that there is no switching frequency parameter in the conventional averaged model. Therefore, this conventional averaged model cannot be used to analyze the influence of the switching frequency on the low-frequency oscillation in the voltage-controlled buck-boost converter.

In $[17,18]$, the duty cycle can be modeled by another method, which contains the switching frequency. For brevity to model the duty cycle $\langle d\rangle,(4.1)$ is rewritten as follows:

$$
\dot{x}=A_{0} x+b_{0}+\left(A_{1} x+b_{1}\right)\langle d\rangle,
$$

where, $x=\left[\left\langle i_{L}\right\rangle,\left\langle v_{0}\right\rangle,\left\langle v_{\mathrm{vf}}\right\rangle\right]^{\mathrm{T}}, \mathrm{T}$ denotes the transpose of the matrix, $A_{0}$ is the coefficient constant matrix of $x, A_{1}$ is the coefficient constant matrix of $x\langle d\rangle, b_{0}$ is constant matrix, and $b_{1}$ is the coefficient constant matrix of $\langle d\rangle$. Their expressions are derived as follows:

$$
\begin{gathered}
A_{0}=\left[\begin{array}{ccc}
0 & \frac{1}{L} & 0 \\
-\frac{1}{C} & -\frac{1}{R C} & 0 \\
-\frac{R_{\mathrm{vf}}}{C\left(2 R_{\mathrm{vi}}+R_{\mathrm{vd}}\right)} & \frac{R C-R_{\mathrm{vf}} C_{\mathrm{vf}}}{\left(2 R_{\mathrm{vi}}+R_{\mathrm{vd}}\right) C_{\mathrm{vf}} R C} & 0
\end{array}\right], \quad b_{0}=\left[\begin{array}{c}
0 \\
0 \\
\frac{V_{\mathrm{ref}}}{C_{\mathrm{vf}} R_{\mathrm{vd}}}
\end{array}\right], \\
A_{1}=\left[\begin{array}{ccc}
0 & -\frac{1}{L} & 0 \\
\frac{1}{C} & 0 & 0 \\
\frac{R_{\mathrm{vf}}}{C\left(2 R_{\mathrm{vi}}+R_{\mathrm{vd}}\right)} & 0 & 0
\end{array}\right], \quad b_{1}=\left[\begin{array}{c}
\frac{V_{\mathrm{in}}}{L} \\
0 \\
0
\end{array}\right] .
\end{gathered}
$$


According to $[17,18]$, the duty cycle $\langle d\rangle$ can be described as follows:

$$
K x+\frac{K}{2 f}\left[A_{1} x+b_{1}\right]\left[\langle d\rangle-\langle d\rangle^{2}\right]=V_{m}\langle d\rangle,
$$

where $K=[0,0,1]$.

By solving (4.4), the following equation can be obtained:

$$
\langle d\rangle^{2}+\left(\frac{a V_{m}}{\left\langle i_{L}\right\rangle}-1\right)\langle d\rangle-\frac{a\left\langle v_{\mathrm{vf}}\right\rangle}{\left\langle i_{L}\right\rangle}=0,
$$

where $a=2 f C\left(2 R_{\mathrm{vi}}+R_{\mathrm{vd}}\right) / R_{\mathrm{vf}}$.

Thus, the duty cycle $\langle d\rangle$ is

$$
\langle d\rangle=\frac{1}{2}-\frac{a V_{m}}{2\left\langle i_{L}\right\rangle}+\sqrt{\left(\frac{1}{2}-\frac{a V_{m}}{2\left\langle i_{L}\right\rangle}\right)^{2}+\frac{a\left\langle v_{\mathrm{vf}}\right\rangle}{\left\langle i_{L}\right\rangle}} .
$$

From (4.6), it is easy to see that the switching frequency is included apparently. Substituting (4.6) into (4.1), the improved averaged model of the system can be derived,

$$
\begin{gathered}
\frac{d\left\langle i_{L}\right\rangle}{d t}=\frac{\left\langle v_{0}\right\rangle+V_{\mathrm{in}}}{2 L}-\frac{a V_{m}\left(V_{\mathrm{in}}-\left\langle v_{0}\right\rangle\right)}{2 L\left\langle i_{L}\right\rangle}+\frac{V_{\mathrm{in}}-\left\langle v_{0}\right\rangle}{L} g\left(\left\langle i_{L}\right\rangle,\left\langle v_{\mathrm{vf}}\right\rangle\right), \\
\frac{d\left\langle v_{0}\right\rangle}{d t}=-\frac{\left\langle i_{L}\right\rangle}{2 C}-\frac{\left\langle v_{0}\right\rangle}{R C}-\frac{a V_{m}}{2 C}+\frac{\left\langle i_{L}\right\rangle}{C} g\left(\left\langle i_{L}\right\rangle,\left\langle v_{\mathrm{vf}}\right\rangle\right), \\
\frac{d\left\langle v_{\mathrm{vf}}\right\rangle}{d t}=\frac{R_{\mathrm{vf}}}{2 R_{\mathrm{vi}}+R_{\mathrm{vd}}}\left(-\frac{\left\langle i_{L}\right\rangle}{2 C}-\frac{a V_{m}}{2 C}+\frac{\left\langle i_{L}\right\rangle}{C} g\left(\left\langle i_{L}\right\rangle,\left\langle v_{\mathrm{vf}}\right\rangle\right)+\frac{\left\langle v_{0}\right\rangle}{C_{\mathrm{vf}} R_{\mathrm{vf}}}-\frac{\left\langle v_{0}\right\rangle}{R C}\right)+\frac{V_{\mathrm{ref}}}{C_{\mathrm{vf}} R_{\mathrm{vd}}},
\end{gathered}
$$

where

$$
g\left(\left\langle i_{L}\right\rangle,\left\langle v_{\mathrm{vf}}\right\rangle\right)=\sqrt{\left(\frac{1}{2}-\frac{a V_{m}}{2\left\langle i_{L}\right\rangle}\right)^{2}+\frac{a\left\langle v_{\mathrm{vf}}\right\rangle}{\left\langle i_{L}\right\rangle}} .
$$

Since $g\left(\left\langle i_{L}\right\rangle,\left\langle v_{\mathrm{vf}}\right\rangle\right)$ contains the parameter $a$, which contains the switching frequency $f$. Therefore, the improved averaged model, that is (4.7), depends on switching frequency $f$. Furthermore, the stability and the bifurcation type of (4.7) can be identified by observing the calculated eigenvalues of its Jacobian matrix at equilibrium point. The equilibrium point of 
(4.7) is as follows:

$$
\begin{gathered}
\left\langle V_{0}\right\rangle=-\frac{2 R_{\mathrm{vi}}+R_{\mathrm{vd}}}{R_{\mathrm{vd}}} V_{\text {ref }}, \\
\left\langle I_{L}\right\rangle=\frac{\left\langle V_{0}\right\rangle^{2}-V_{\mathrm{in}}\left\langle V_{0}\right\rangle}{R V_{\text {in }}}, \\
\left\langle V_{\mathrm{vf}}\right\rangle=\frac{\left\langle I_{L}\right\rangle}{a}\left(1+\frac{\left\langle V_{0}\right\rangle}{R\left\langle I_{L}\right\rangle}\right)^{2}+\left(V_{m}-\frac{\left\langle I_{L}\right\rangle}{a}\right)\left(1+\frac{\left\langle V_{0}\right\rangle}{R\left\langle I_{L}\right\rangle}\right), \\
\langle D\rangle=\frac{\left(2 R_{\mathrm{vi}}+R_{\mathrm{vd}}\right) V_{\mathrm{ref}}}{\left(2 R_{\mathrm{vi}}+R_{\mathrm{vd}}\right) V_{\mathrm{ref}}+V_{\mathrm{in}} R_{\mathrm{vd}}} .
\end{gathered}
$$

Note that $\left\langle I_{L}\right\rangle,\left\langle V_{0}\right\rangle,\left\langle V_{\mathrm{vf}}\right\rangle$, and $\langle D\rangle$ are the steady state value of $\left\langle i_{L}\right\rangle,\left\langle v_{0}\right\rangle,\left\langle v_{\mathrm{vf}}\right\rangle$, and $\langle d\rangle$, respectively. From (4.9)-(4.12), it is found that the steady state values $\left\langle V_{0}\right\rangle,\left\langle I_{L}\right\rangle$, and $\langle D\rangle$ do not depend on the switching frequency. However, the steady state value $\left\langle V_{\mathrm{vf}}\right\rangle$ contains the switching frequency parameter, and it leads its value to be changed with the switching frequency changing.

Additionally, since the CCM operation in the voltage-controlled buck-boost converter is only concerned in this paper, the duty cycle constraint must be considered. According to $[1]$, the condition for CCM operation is as follows:

$$
\frac{L f}{R} \geq \frac{(1-\langle D\rangle)^{2}}{2}
$$

So, the duty cycle constraint is as follows:

$$
\langle D\rangle \geq 1-\sqrt{\frac{2 L f}{R}} .
$$

Furthermore, substituting (4.12) into (4.14), the switching frequency under CCM operation must satisfy the following inequality:

$$
f \geq \frac{R V_{\mathrm{in}}^{2} R_{\mathrm{vd}}^{2}}{2 L\left(V_{\mathrm{in}} R_{\mathrm{vd}}+2 R_{\mathrm{vi}} V_{\text {ref }}+R_{\mathrm{vd}} V_{\text {ref }}\right)^{2}} .
$$

Also, the Jacobian matrix $A_{g a}$ for (4.7) at its equilibrium point can be calculated as follows:

$$
A_{g a}=\left[\begin{array}{ccc}
\frac{\left(V_{\mathrm{in}}-\left\langle V_{0}\right\rangle\right)\left(2 g_{1}\left\langle I_{L}\right\rangle^{2}+a V_{m}\right)}{2 L\left\langle I_{L}\right\rangle^{2}} & -\frac{g_{0}}{L}+\frac{1}{2 L}+\frac{a V_{m}}{2 L\left\langle I_{L}\right\rangle} & \frac{\left(V_{\mathrm{in}}-\left\langle V_{0}\right\rangle\right) g_{2}}{L} \\
\frac{2 g_{0}-1}{2 C}+\frac{\left\langle I_{L}\right\rangle g_{1}}{C} & -\frac{1}{R C} & \frac{\left\langle I_{L}\right\rangle g_{2}}{C} \\
\frac{R_{\mathrm{vf}}\left(2 g_{0}-1+2\left\langle I_{L}\right\rangle g_{1}\right)}{2\left(2 R_{\mathrm{vi}}+R_{\mathrm{vd}}\right) C} & \frac{R C-C_{\mathrm{vf}} R_{\mathrm{vf}}}{R C\left(2 R_{\mathrm{vi}}+R_{\mathrm{vd}}\right) C_{\mathrm{vf}}} & \frac{R_{\mathrm{vf}}\left\langle I_{L}\right\rangle g_{2}}{\left(2 R_{\mathrm{vi}}+R_{\mathrm{vd}}\right) C}
\end{array}\right],
$$


where

$$
\begin{gathered}
g_{0}=\left.g\left(\left\langle i_{L}\right\rangle,\left\langle v_{\mathrm{vf}}\right\rangle\right)\right|_{\substack{\left\langle i_{L}\right\rangle=\left\langle I_{L}\right\rangle \\
\left\langle v_{\mathrm{vf}}\right\rangle=\left\langle V_{\mathrm{vf}}\right\rangle}}=\sqrt{\left(\frac{1}{2}-\frac{a V_{m}}{2\left\langle I_{L}\right\rangle}\right)^{2}+\frac{a\left\langle V_{\mathrm{vf}}\right\rangle}{\left\langle I_{L}\right\rangle},} \\
g_{1}=\left.\frac{\partial g\left(\left\langle i_{L}\right\rangle,\left\langle v_{\mathrm{vf}}\right\rangle\right)}{\partial\left\langle i_{L}\right\rangle}\right|_{\substack{\left\langle i_{L}\right\rangle=\left\langle I_{L}\right\rangle \\
\left\langle v_{\mathrm{vf}}\right\rangle=\left\langle V_{\mathrm{vf}}\right\rangle}}=\frac{1}{2}\left(\left(\frac{1}{2}-\frac{a V_{m}}{2\left\langle I_{L}\right\rangle}\right)^{2}+\frac{a\left\langle V_{\mathrm{vf}}\right\rangle}{\left\langle I_{L}\right\rangle}\right)^{-1 / 2}\left(\left(\frac{1}{2}-\frac{a V_{m}}{2\left\langle I_{L}\right\rangle}\right) \frac{a V_{m}}{\left\langle I_{L}\right\rangle^{2}}-\frac{a\left\langle V_{\mathrm{vf}}\right\rangle}{\left\langle I_{L}\right\rangle^{2}}\right), \\
g_{2}=\left.\frac{\partial g\left(\left\langle i_{L}\right\rangle,\left\langle v_{\mathrm{vf}}\right\rangle\right)}{\partial\left\langle v_{\mathrm{vf}}\right\rangle}\right|_{\substack{\left\langle i_{L}\right\rangle=\left\langle I_{L}\right\rangle \\
\left\langle v_{\mathrm{vf}}\right\rangle=\left\langle V_{\mathrm{vf}}\right\rangle}}=\frac{a}{2\left\langle I_{L}\right\rangle}\left(\left(\frac{1}{2}-\frac{a V_{m}}{2\left\langle I_{L}\right\rangle}\right)^{2}+\frac{a\left\langle V_{\mathrm{vf}}\right\rangle}{\left\langle I_{L}\right\rangle}\right)^{-1 / 2} .
\end{gathered}
$$

According to [13], we know that if all the eigenvalues of Jacobian matrix lay in the left of the complex plane, the system is in stable operation. But, if there is a pair of complex eigenvalues across the imaginary axis to the right of the complex plane, it implies that Hopf bifurcation occurs in the system.

\section{Influence Analysis}

The circuit parameters are shown in Section 3. From (4.15), the switching frequency must be larger than $2.076 \mathrm{kHz}$ to maintain the voltage-controlled buck-boost converter operating in CCM operation. Based on (4.16), the eigenvalues at the different switching frequency are calculated and noted down in Table 1. It is found that the system is in stable operation when $f \geq 14.8 \mathrm{kHz}$ since all the eigenvalues lay in the left of the complex plane. Therefore, the voltage-controlled buck-boost converter must be in stable operation when $f=20 \mathrm{kHz}$, which has already been shown in Figure 3.

But, when the switching frequency is equal to $14.7 \mathrm{kHz}$, that is, $f=14.7 \mathrm{kHz}$, Hopf bifurcation occurs since there is a pair of complex eigenvalues across the imaginary axis to the right of the complex plane. Therefore, the system will operate in unstable operation. However, due to the topology of voltage-controlled buck-boost converter and the characteristic of the diode, the inductor current cannot also be enlarged on and on. Thus, the output voltage $v_{0}$ cannot also be enlarged on and on. Finally, the low-frequency oscillation is formed. Therefore, the voltage-controlled buck-boost converter must be in low-frequency oscillation operation when $f=4 \mathrm{kHz}$, which has already been shown in Figure 4 .

Additionally, by noting the bifurcation points at the different parameter spaces, the stable boundaries can also be obtained and shown in Figures 7 and 8 . It is found that the results between the theoretical analysis and MATLAB/Simulink simulations are in good agreement with each other. So, it is effective to establish the improved averaged model to analyze the influence of switching frequency on the low-frequency oscillation in the voltagecontrolled buck-boost converter. 
Table 1: The eigenvalues of Jacobian matrix at the different switching frequency $f$.

\begin{tabular}{lccc}
\hline$f$ & $\lambda_{1,2}$ & $\lambda_{3}$ & State \\
\hline $150.0 \mathrm{kHz}$ & $-50.398633 \pm 3085.4313 i$ & -38.155568 & Stable \\
$100.0 \mathrm{kHz}$ & $-47.624760 \pm 3083.5781 i$ & -38.186625 & Stable \\
$50.0 \mathrm{kHz}$ & $-39.316753 \pm 3078.0066 i$ & -38.280093 & Stable \\
$20.0 \mathrm{kHz}$ & $-14.514534 \pm 3061.1835 i$ & -38.563187 & Stable \\
$14.8 \mathrm{kHz}$ & $-0.0749087 \pm 3051.2569 i$ & -38.730860 & Stable \\
$14.7 \mathrm{kHz}$ & $0.30206437 \pm 3050.9964 i$ & -38.735267 & Unstable (Hopf bifurcation) \\
\hline
\end{tabular}

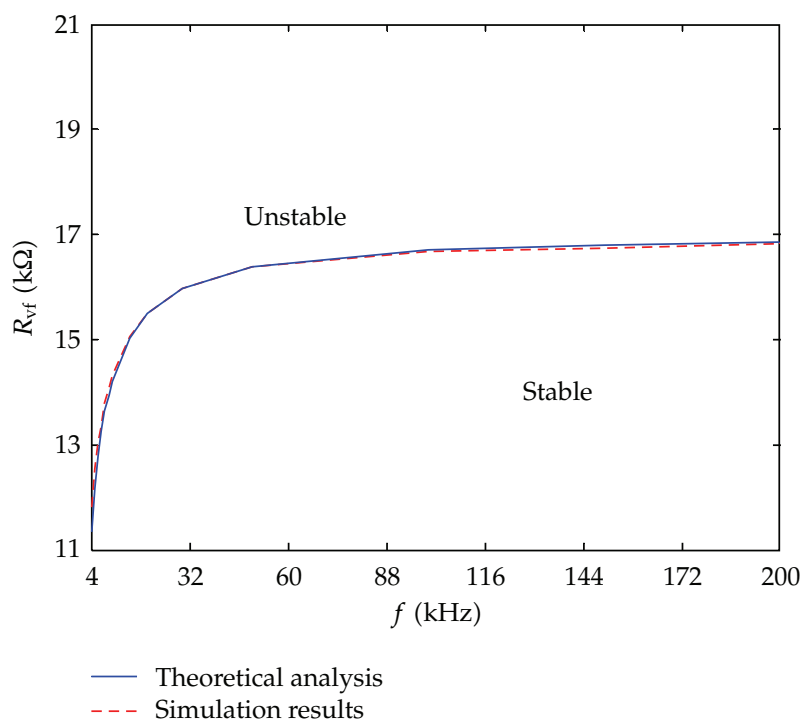

Figure 7: Comparison of the theoretical analysis with simulation results in the parameter space of $f$ versus $R_{\mathrm{vf}}$.

\section{Circuit Experiment}

In order to confirm the influence of the switching frequency on the low-frequency oscillation in the voltage-controlled buck-boost converter and corresponding theoretical analysis, the circuit of the voltage-controlled buck-boost converter is designed and its full schematic diagram with detailed specifications is depicted in Figure 9. In our experiment, the voltage probe Agilent $10074 \mathrm{C}$ is used to detect the output voltage $v_{0}$, the current probe Tektronix A622 is used to detect the inductor current $i_{L}$ and the digital oscilloscope Agilent 54622D is employed to capture the measured waveforms from the probes. Moreover, the switch $Q$ is realized by IRFP460, the diode $D$ is realized by MUR1560, the amplifiers $\mathrm{AM}_{1}$ and $\mathrm{AM}_{2}$ are realized by LF356, and the comparator COM is realized by LM311. Additionally, the photocoupler TLP250 should be inserted for driving the switch IRFP460 since the ground of IRFP460 is floating. Additionally, in our experiment, it is also noted that

(1) $V_{\mathrm{cc}}=15 \mathrm{~V}, V_{D}=18 \mathrm{~V}, R_{T 1}=1.5 \mathrm{k} \Omega, R_{T 2}=268 \Omega, R_{T 3}=510 \Omega, R_{D}=510 \Omega$, and other circuit parameters' value are shown in Section 3; 


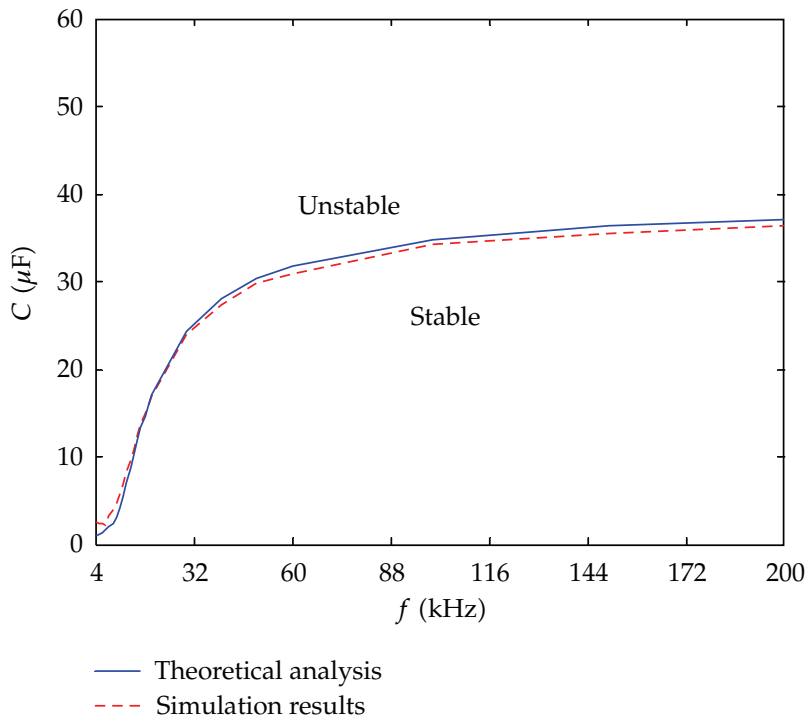

Figure 8: Comparison of the theoretical analysis with simulation results in the parameter space of $f$ versus C.

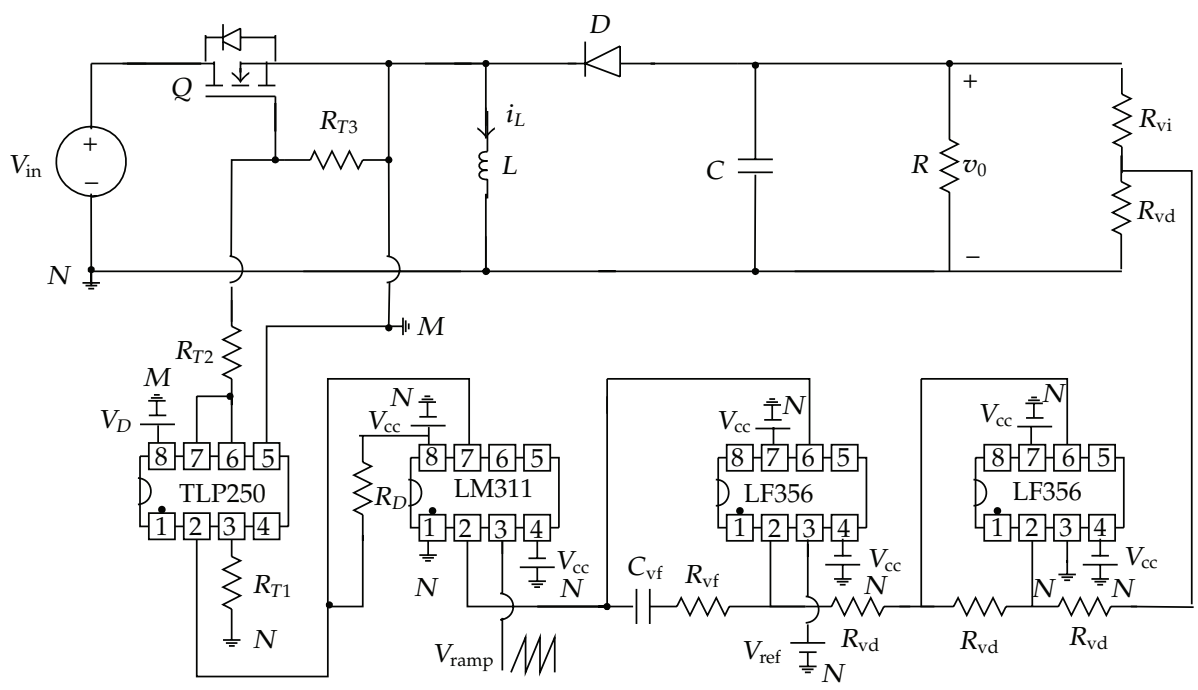

Figure 9: Full schematic diagram of the experimental circuit.

(2) there are two types of ground in the TLP250. The first is $N$, which is the same as LM311 and LF356; however, the second is $M$, which is different from $N$ and it is essential to remain isolated.

As calculated in Section 5, the voltage-controlled buck-boost converter is in stable operation when $f \geq 14.8 \mathrm{kHz}$. Otherwise, the system will operate in low-frequency oscillation. Figure 10 shows the experimental results for $f=20 \mathrm{kHz}$, which is above the critical point. It is obvious that the system is in stable operation. 


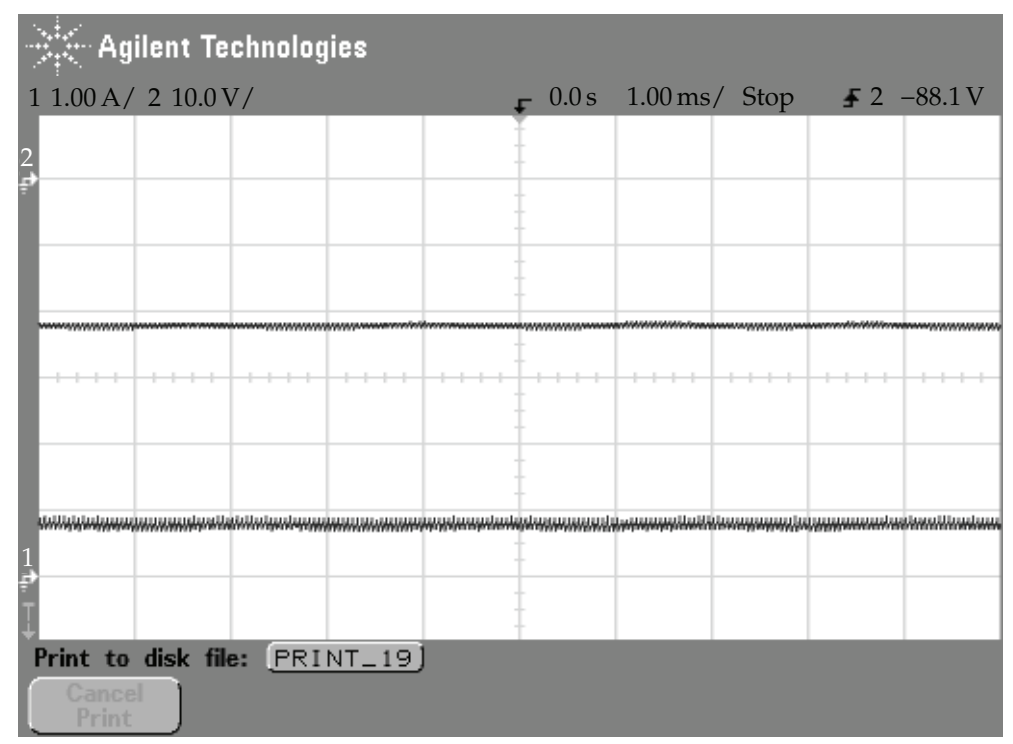

Figure 10: Measured waveforms for $f=20 \mathrm{kHz}$ : the voltage $v_{0}$ (upper trace: $10 \mathrm{~V} / \mathrm{div}$ ) and the inductor current $i_{L}$ (lower trace: $1 \mathrm{~A} / \mathrm{div}$ ), time scale: $1 \mathrm{~ms} / \mathrm{div}$.

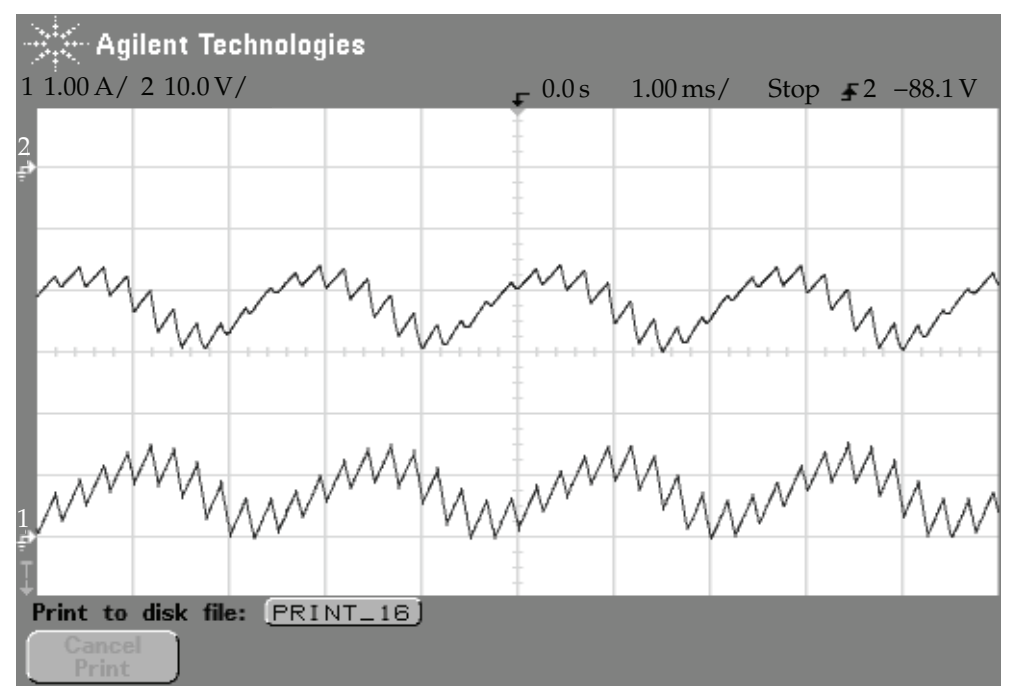

Figure 11: Measured waveforms for $f=4 \mathrm{kHz}$ : the voltage $v_{0}$ (upper trace: $10 \mathrm{~V} / \mathrm{div}$ ) and the inductor current $i_{L}$ (lower trace: $1 \mathrm{~A} / \mathrm{div}$ ), time scale: $1 \mathrm{~ms} / \mathrm{div}$.

However, when we choose the switching frequency equal to $4 \mathrm{kHz}$, that is, $f=4 \mathrm{kHz}$, the voltage-controlled buck-boost converter really operates in low-frequency oscillation operation, which is shown in Figure 11. Furthermore, comparing the circuit experimental results (Figures 10 and 11) with the simulation results (Figures 3 and 4), it is found that they are in good agreement with each other qualitatively. The discrepancy is mainly caused by the fact that all the circuit components are considered as ideal in simulation. 


\section{Conclusion}

Based on the established improved averaged model, the influence of switching frequency on the low-frequency oscillation in the voltage-controlled buck-boost converter is analyzed in this paper. The MATLAB/Simulink simulation results, theoretical results, and circuit experimental results described that the switching frequency has an important influence on the low-frequency oscillation in the voltage-controlled buck-boost converter, that is, the lowfrequency oscillation is easy to occur when the switching frequency is low. Additionally, by comparing the conventional averaged model, the improved averaged model is an effective model to analyze the dynamical behaviors of the voltage-controlled buck-boost converter under different switching frequency.

\section{Acknowledgments}

This paper was supported by National Natural Science Foundation of China (Grant no. 51007068), Specialized Research Fund for the Doctoral Program of Higher Education (Grant no. 20100201120028), the Fundamental Research Funds for the Central Universities, and the State Key Laboratory of Electrical Insulation and Power Equipment (Grant no. EIPE10303).

\section{References}

[1] M. K. Kazimierczuk, Pulse-Width Modulated DC-DC Power Converters, John Wiley \& Sons, Oxford, UK, 2008.

[2] K. C. Wu, "A comprehensive analysis of current-mode control for DCM buck-boost converters," IEEE Transactions on Industrial Electronics, vol. 51, no. 3, pp. 733-735, 2004.

[3] A. E. Aroudi, L. Benadero, E. Toribio, and S. Machiche, "Quasiperiodicity and chaos in the DC-DC Buck-Boost converter," International Journal of Bifurcation and Chaos in Applied Sciences and Engineering, vol. 10, no. 2, pp. 359-371, 2000.

[4] J. Wu, M. Liu, and P. Yang, "Study of bifurcation and chaos in the current-mode controlled Buck-Boost DC-DC converter (I)-modeling and simulation," Control Theory and Applications, vol. 19, no. 3, pp. 387-394, 2002.

[5] J. Wu, M. Liu, and P. Yang, "Study of bifurcation and chaos in the current-mode controlled Buck-Boost DC-DC converter (II)-numerical analysis and experiment," Control Theory and Applications, vol. 19, no. 3, pp. 395-401, 2002.

[6] D. Negoitescu, D. Lascu, V. Popescu, and C. Ivan, "Bifurcation and chaotic aspects in peak current controlled buck-boost converters," WSEAS Transactions on Circuits and Systems, vol. 7, no. 7, pp. 688697, 2008.

[7] K. W. E. Cheng, M. Liu, and J. Wu, "Chaos study and parameter-space analysis of the DC-DC buckboost converter," IEE Proceedings, vol. 150, no. 2, pp. 126-138, 2003.

[8] D. Dai, Y. Ma, C. K. Tse, and X. Ma, "Existence of horseshoe maps in current-mode controlled buckboost dc/dc converters," Chaos, Solitons E Fractals, vol. 25, no. 3, pp. 549-556, 2005.

[9] K. W. E. Cheng, M. Liu, and Y. L. Ho, "Experimental confirmation of frequency correlation for bifurcation in current-mode controlled buck-boost converters," IEEE Power Electronics Letters, vol. 1, no. 4, pp. 101-103, 2003.

[10] K. W. E. Cheng, M. Liu, and J. Wu, "Experimental study of bifurcation and chaos in the Buck-Boost converter," IEE Proceedings, vol. 150, no. 1, pp. 45-61, 2003.

[11] C. Xu, K. W. E. Cheng, Z. H. Shi, and K. Ding, "Nonlinear phenomena of the buck-boost converter analyzed by storage energy," in Proceedings of the 3rd International Conference on Power Electronics Systems and Applications (PESA '09), 2009.

[12] F. Wang, H. Zhang, and X. Ma, "Analysis of slow-scale instability in boost pfc converter using the method of harmonic balance and floquet theory," IEEE Transactions on Circuits and Systems I, vol. 57, no. 2, pp. 405-414, 2010. 
[13] H. H. C. Iu and C. K. Tse, "Study of low-frequency bifurcation phenomena of a parallel-connected boost converter system via simple averaged models," IEEE Transactions on Circuits and Systems I, vol. 50, no. 5, pp. 679-686, 2003.

[14] J. H. Su, C. L. Chien, J. J. Chen, and C. M. Wang, "Simulink behavior models for DC-DC switching converter circuits using PWM control ICs," International Journal of Engineering Education, vol. 22, no. 2, pp. 315-322, 2006.

[15] V. F. Pires and J. F. A. Silva, “Teaching nonlinear modeling, simulation, and control of electronic power converters using MATLAB/SIMULINK," IEEE Transactions on Education, vol. 45, no. 3, pp. 253-261, 2002.

[16] J. Vadillo, J. M. Echeverria, M. Martinez-Iturralde, and L. Fontan, "Modelling and simulation of indirect space vector modulated matrix converter using MATLAB/Simulink," International Journal of Electronics, vol. 96, no. 8, pp. 855-863, 2009.

[17] B. Lehman and R. M. Bass, "Extensions of averaging theory for power electronic systems," IEEE Transactions on Power Electronics, vol. 11, no. 4, pp. 542-553, 1996.

[18] B. Lehman and R. M. Bass, "Switching frequency dependent averaged models for PWM DC-DC converters," IEEE Transactions on Power Electronics, vol. 11, no. 1, pp. 89-98, 1996. 


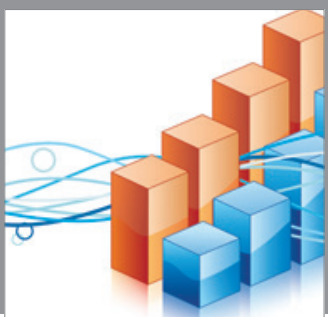

Advances in

Operations Research

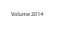

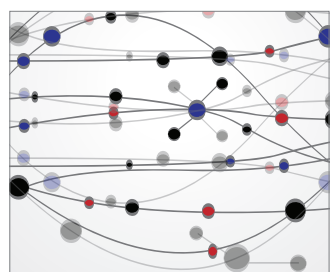

\section{The Scientific} World Journal
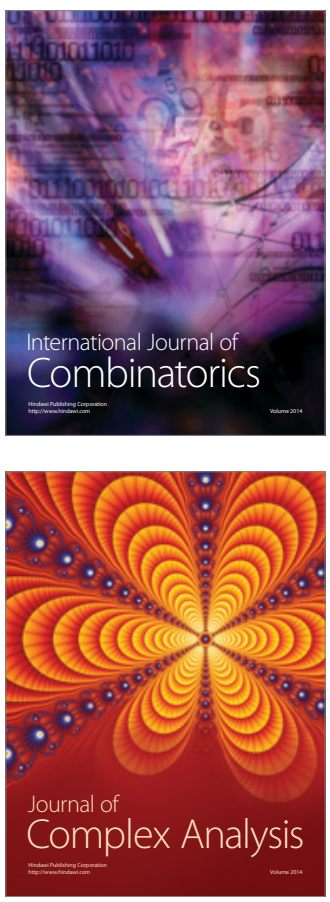

International Journal of

Mathematics and

Mathematical

Sciences
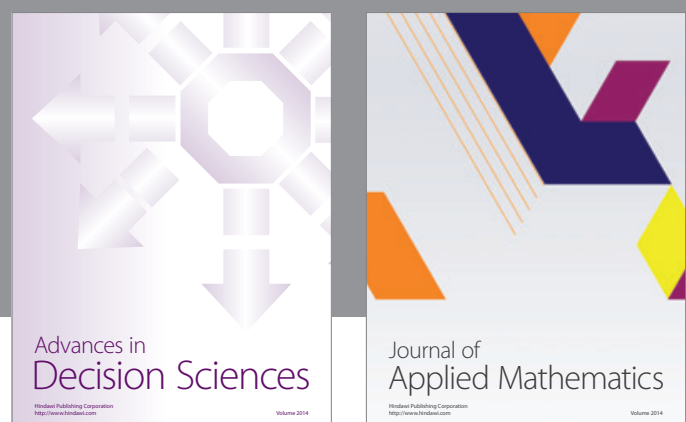

Journal of

Applied Mathematics
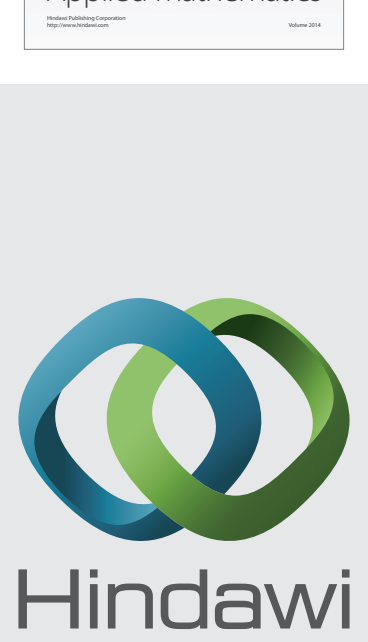

Submit your manuscripts at http://www.hindawi.com
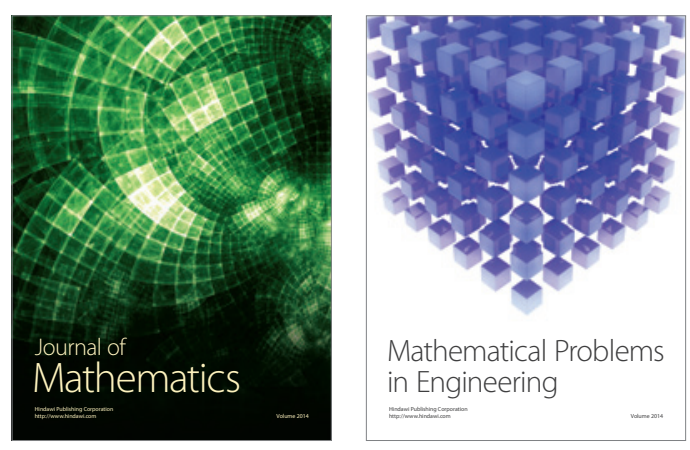

Mathematical Problems in Engineering
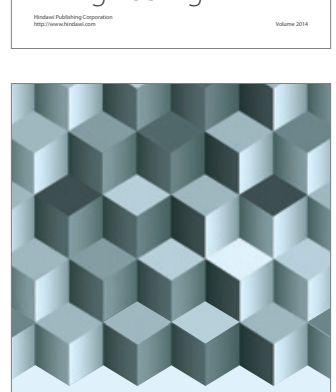

Journal of

Function Spaces
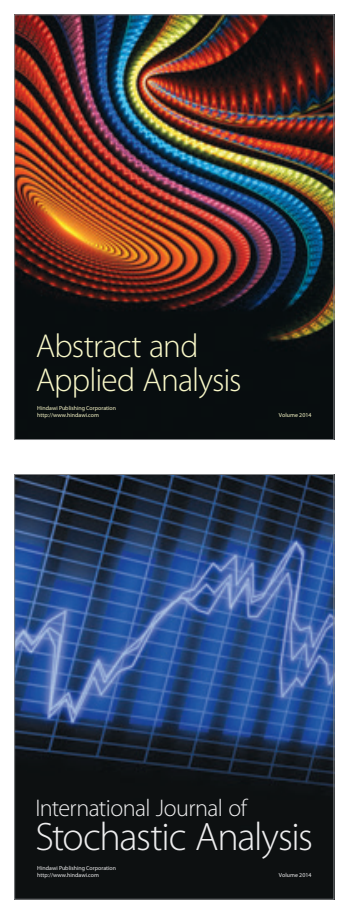

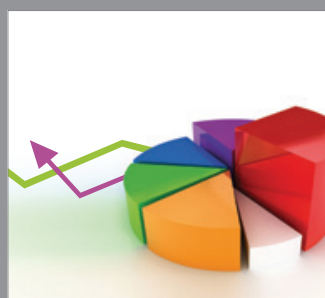

ournal of

Probability and Statistics

Promensencen
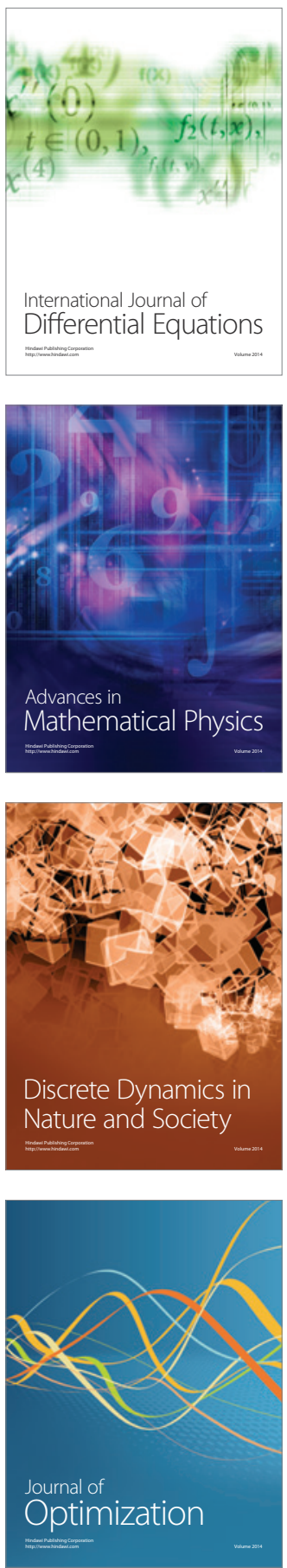